\title{
EDITORIAL
}

\section{Epigenetics in comparative physiology}

\author{
Hans H. Hoppeler*
}

Since the earliest definition of the term 'epigenetics' in 1942 (Waddington, 1942), our understanding of the concept has been refined extensively. C. H. Waddington initially understood epigenetics as the mechanism by which genes give rise to the phenotype during development (Waddington, 1957). However, we now see epigenetics more broadly as mitotically or meiotically heritable changes in gene function that are not due to changes in the DNA sequence. Adrian Bird placed epigenetics in an organismal and functional context when he defined it as, 'The structural adaptation of chromosomal regions so as to register, signal or perpetuate altered activity states' (Bird, 2007). While epigenetic phenomena have far-reaching implications for adaptation and evolution, they are regulated at the level of the gene through various molecular mechanisms, including DNA methylation, RNA interference and histone modification. In addition, epigenetically induced states of altered gene expression can persist for variable time spans within a cell's life, across mitotic divisions and even across meiosis, and are ultimately responsible for the phenomenon of cell memory.

The rapid expansion in recent understanding that epigenetic mechanisms mediate the interaction between the genome and the environment in phenotypic plasticity and evolution is one of the many reasons why The Journal of Experimental Biology has chosen the theme of 'epigenetics' for this special issue. While barely more than 100 manuscripts were published containing the search term 'epigenetic' in 1995, the number of publications on the subject has grown to over 6500 in 2014. And as many comparative physiologists are driven by the desire to understand the evolution of the physiological traits that are seen today, we have taken the opportunity to introduce the concepts that are central to epigenetics in a general context and also from a comparative perspective in this collection of reviews.

In his introduction, Noble (Noble, 2015) discusses how epigenetic effects - in particular trans-generational inheritance contradict the core concepts of the Modern Neo-Darwinian Synthesis. He postulates that the reductionist gene-centered theory of evolution needs to be replaced by a more comprehensive 'Integrative Synthesis' including additional mechanisms, such as the inheritance of acquired characteristics. The fact that environmental factors can strongly modify gene expression through epigenetic mechanisms is also an important aspect of the interindividual variability that is clearly evident in much of the physiological data that are interpreted by comparative experimentalists (Burggren, 2014). In his current article (Burggren, 2015), Burggren goes on to point out that matters are complicated further by the fact that epigenetic modification of a phenotype can change over time - either washing-out or washing-in - in individuals or across generations. This is of practical significance for comparative physiologists who investigate adaptation and evolution at either the individual or population level.

Comparative research also provides some of the most stunning examples of the extent to which epigenetic modifications can change the physical and behavioral attributes of an organism. In social insects, polyphenism - where a single genotype is expressed differently in specific contexts to give rise to discrete phenotypes is held to be responsible for the development of distinct castes. It is also becoming evident that epigenetic mechanisms are responsible for the dramatic physiological adjustments that accompany the switch between the 'well-behaved' solitary locust morph and the devastating gregarious form (Ernst et al., 2015). This phase transition can take hours to develop and generations to subside much in line with the epigenetic wash-in and wash-out phenomena described by Burggren. Bonasio also suggests that epigenetic phenomena may stabilize specific appropriate phenotypes encoded in a single genome in the short term, and potentially over generations, by sexual reproduction (Bonasio, 2015). He maintains, however, that epigenetic states are inherently metastable and that, for inheritance of acquired traits over evolutionary time scales, communication between the soma and the germline needs to be established. He therefore proposes that epigenetic phenomena should be seen within the concept of the 'Modern Synthesis'.

The contradictory viewpoints highlighted in this special issue indicate that the role of epigenetics in evolution is yet to be firmly established. While some are of the opinion that evolutionary theory requires an urgent rethink (Laland et al., 2014), others (Wray et al., 2014) maintain that the Modern Synthesis can accommodate this new expanded approach. Whatever the eventual outcome, the role of epigenetics in evolution will continue to be hotly debated, both from a practical perspective and for academic interest.

\section{References}

Bird, A. (2007). Perceptions of epigenetics. Nature 447, 396-398.

Bonasio, R. (2015). The expanding epigenetic landscape of non-model organisms. J. Exp. Biol. 218, 114-122.

Burggren, W. W. (2014). Epigenetics as a source of variation in comparative animal physiology - or - Lamarck is lookin' pretty good these days. J. Exp. Biol. 217, 682689.

Burggren, W. W. (2015). Dynamics of epigenetic phenomena: inter- and intragenerational phenotype 'washout'. J. Exp. Biol. 218, 80-87.

Ernst, U. R., Van Hiel, M. B., Depuydt, G., Boerjan, B., De Loof, A. and Schoofs, L. (2015). Epigenetics and locust life phase transitions. J. Exp. Biol. 218, 88-99.

Laland, K., Uller, T., Feldman, M., Sterelny, K., Müller, G. B., Moczek, A., Jablonka, E., Odling-Smee, J., Wray, G. A., Hoekstra, H. E. et al. (2014). Does evolutionary theory need a rethink? Yes, urgently. Nature 514, 161-164.

Noble, D. (2015). Evolution beyond neo-Darwinism: a new conceptual framework. J. Exp. Biol. 218, 7-13.

Waddington, C. H. (1942). The epigenotype. Endeavor 1, 18-20.

Waddington, C. H. (1957). The Strategy of the Genes. London: Allen and Unwin.

Wray, G. A., Futuyma, D. A., Lenski, R. E., MacKay, T. F. C., Schluter, D., Strassman, J. E. and Hoekstra, H. E. (2014). Does evolutionary biology need a rethink? No, all is well. Nature 514, 161-164. 